http://jmscr.igmpublication.org/home/

ISSN (e)-2347-176x ISSN (p) 2455-0450

crossref DOI: https://dx.doi.org/10.18535/jmscr/v7i10.105

Journal Of Medical Science And Clinical Research

IGM Publication

An Official Publication of IGM Publication

\title{
An Observational Study on Serum Ferritin Level in Dengue Fever and Its Correlation with Severity of Dengue Fever
}

\author{
Authors
}

\section{K. Gokul Bharathi, R Aswinth, S. Sudarshan}

\begin{abstract}
Aims and Objectives: To study the serum ferritin level in dengue fever and its correlation with severity of dengue fever

Methods: The study was performed on patients admitted for dengue fever in Rajah Muthiah Medical College and Hospital under the Division of General Medicine from October 2017 to September 2019.After satisfying the inclusion and exclusion criteria 50 dengue IgM positive patients were included in this study. Their serum ferritin levels on the day of admission was measured. The relationship between serum ferritin levels and platelets and severity of dengue fever was observed

Results: There was significant correlation between serum ferritin levels and thrombocytopenia $(p=0.006)$.Patients with increased ferritin levels had significant decrease in platelet count. Bleeding tendencies were found to be higher in patients who had increased ferritin levels and thrombocytopenia.

Conclusion: Serum ferritin levels were increased in dengue fever. Patients with high ferritin levels were found to have increased incidence of thrombocytopenia. Hence high ferritin levels can be used as a indicator for assessing disease severity and prognosis of dengue fever patients. Those patients with high serum ferritin levels should be monitored carefully.
\end{abstract}

\section{Introduction}

Ferritin is a acute phase reactant produced by reticulo-endothelial cells in response to inflammation and infection. It is produced in significant amount by monocytes, macrophages and hepatic cells. It has been shown that synthesis of ferritin can be induced by cytokines.

The reticuo endothelial system plays a critical role in iron metabolism by processing heamoglobin from senescent red blood cells. Acute inflammation and infection induce the blockade of iron release resulting in a decreased in serum iron, A virulence factor for many microorganisms. Elevated levels of serum ferritin, an acute phase reactant reflect the clinical response to deprive micro organisms of serum iron.
Hyperferritinemia is associated with immune activation and coagulation disturbances. A raise in serum ferritin level has been observed to have linear correlation with severity of dengue fever

\section{Aims and Objectives}

- To measure the serum ferritin levels in dengue fever patients

- To determine the relationship between serum ferritin level and thrombocytopenia in patients with dengue fever

\section{Inclusion Criteria}

Confirmed cases of Dengue IgM positive, IgG positive and NS1 Ag positive (Age > $13 \mathrm{yrs}$ ) 


\section{Exclusion Criteria}

- Pregnancy

- Other febrile illness

- Mixed infections

- Patients with known hematological disorders

- Patients on heparin

- Patients on chemotherapy and radiotherapy / immuno compromised patients.

- Known patients with qualitative and quantitative platelet disorders

\section{Methods}

The study was performed on patients admitted for dengue fever in Rajah Muthiah Medical College and hospital under the Division of General Medicine from October 2017 to September 2019.After satisfying the inclusion and exclusion criteria 50 dengue $\operatorname{IgM}$ positive patients were included in this study. Their serum ferritin levels on the day of admission was measured. The relationship between serum ferritn levels and platelets and severity of dengue fever was observed. Statistical analysis was done using the SPSS software

\section{Patient Characteristics}

Table 1: Sex Distribution of Study Participants

$$
\mathbf{n}=\mathbf{5 0}
$$

\begin{tabular}{|l|c|}
\hline Sex & No. of patients \\
\hline Male & 28 \\
\hline Female & 22 \\
\hline Total & 50 \\
\hline
\end{tabular}

In a total number of 50 patients $56 \%(n=28)$ were males and $44 \%(n=22)$ were females.

Figure 1: Sex Distribution of Study Participants

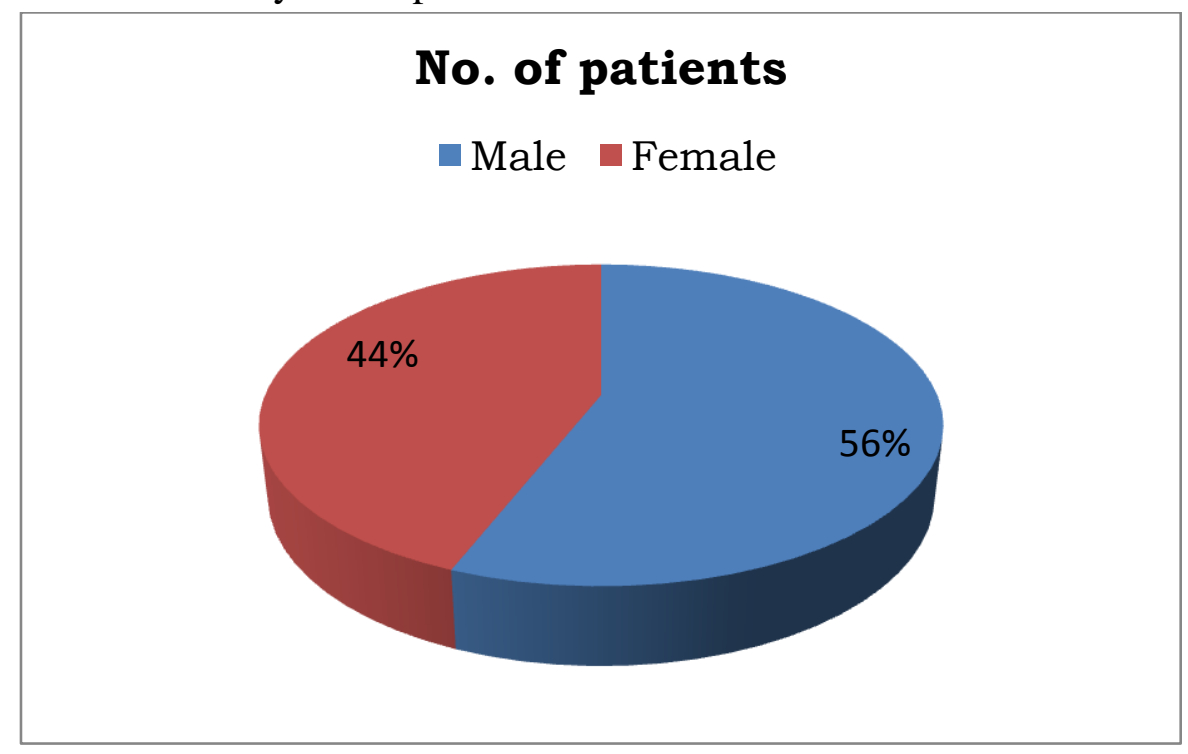

Table 2: Age Distribution of Study Participants

$\mathbf{n}=\mathbf{5 0}$

\begin{tabular}{|l|c|c|}
\hline Age & No. of patients (n) & Percentage (\%) \\
\hline $11-25$ & 19 & 38 \\
\hline $26-40$ & 12 & 24 \\
\hline $41-55$ & 13 & 26 \\
\hline $56-70$ & 2 & 4 \\
\hline $71-85$ & 4 & 8 \\
\hline Total & 50 & 100 \\
\hline
\end{tabular}

In a total number of 50 patients most of them were in the age group of 11-25 $(n=19)$ and least number was in the age group 56-70 $(n=2)$. 
Figure 2: Age Distribution of Study Participants

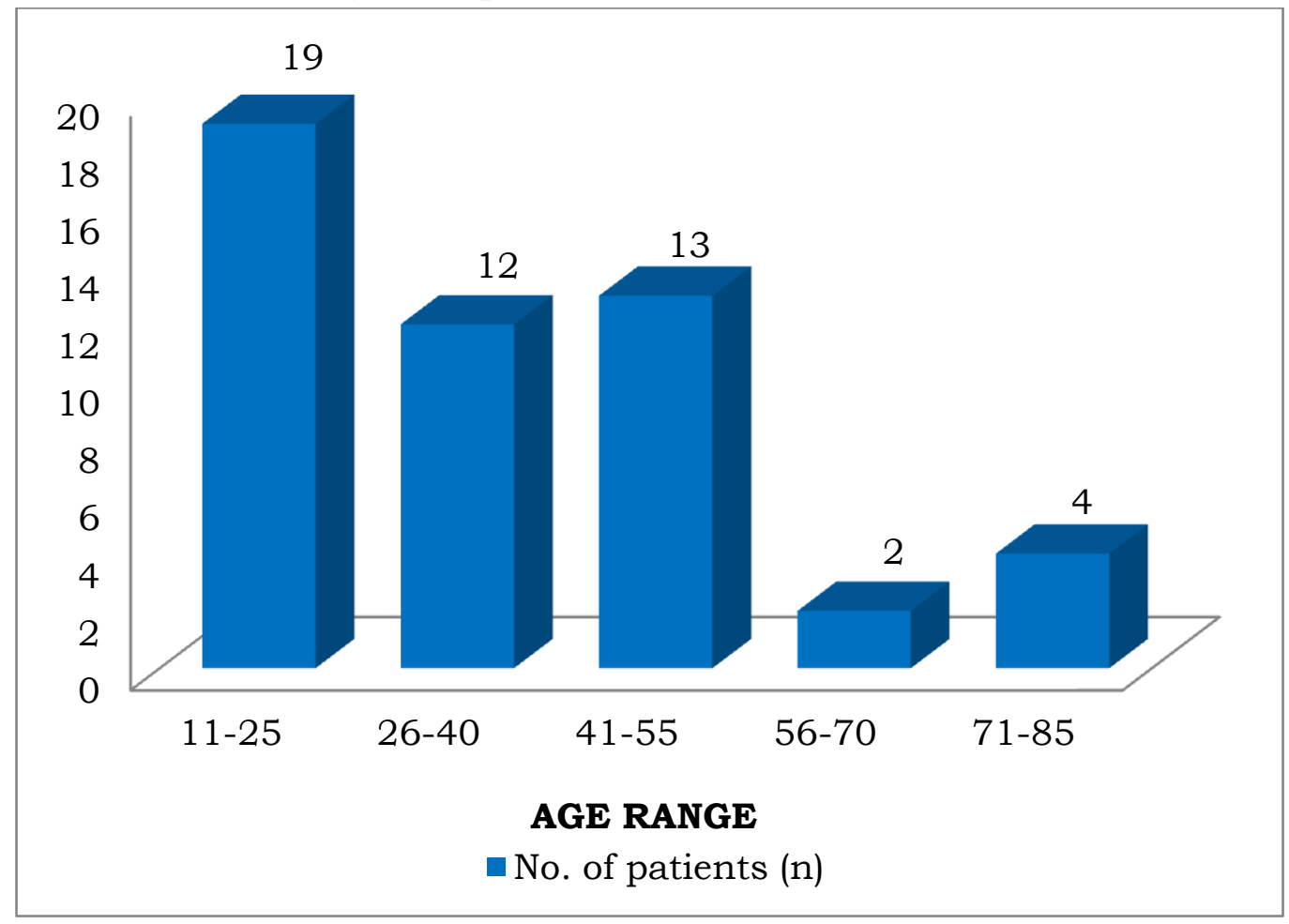

Table 3: Sex Distribution and Ferritin CoRelation among Study Participants

\begin{tabular}{|l|c|c|c|}
\hline \multirow{2}{*}{ SEX } & \multicolumn{2}{|c|}{ FERRITIN } & \multirow{2}{*}{ TOTAL } \\
\cline { 2 - 3 } & HIGH & NORMAL & \\
\hline MALE & 19 & 9 & 28 \\
\hline FEMALE & 11 & 11 & 22 \\
\hline TOTAL & 30 & 20 & 50 \\
\hline
\end{tabular}

Out of 50 patients 28 were male and 22 were female. Chi square test was applied to test the significance between ferritin levels and sex distribution. There was no significant difference ( $\mathrm{p}=.201$ ) found between the variation in ferritin levels and sex distribution. This implies ferritin levels does not depend upon sex distribution.

Figure 3: Sex Distribution of Ferritin Co-Relation among Study Participants

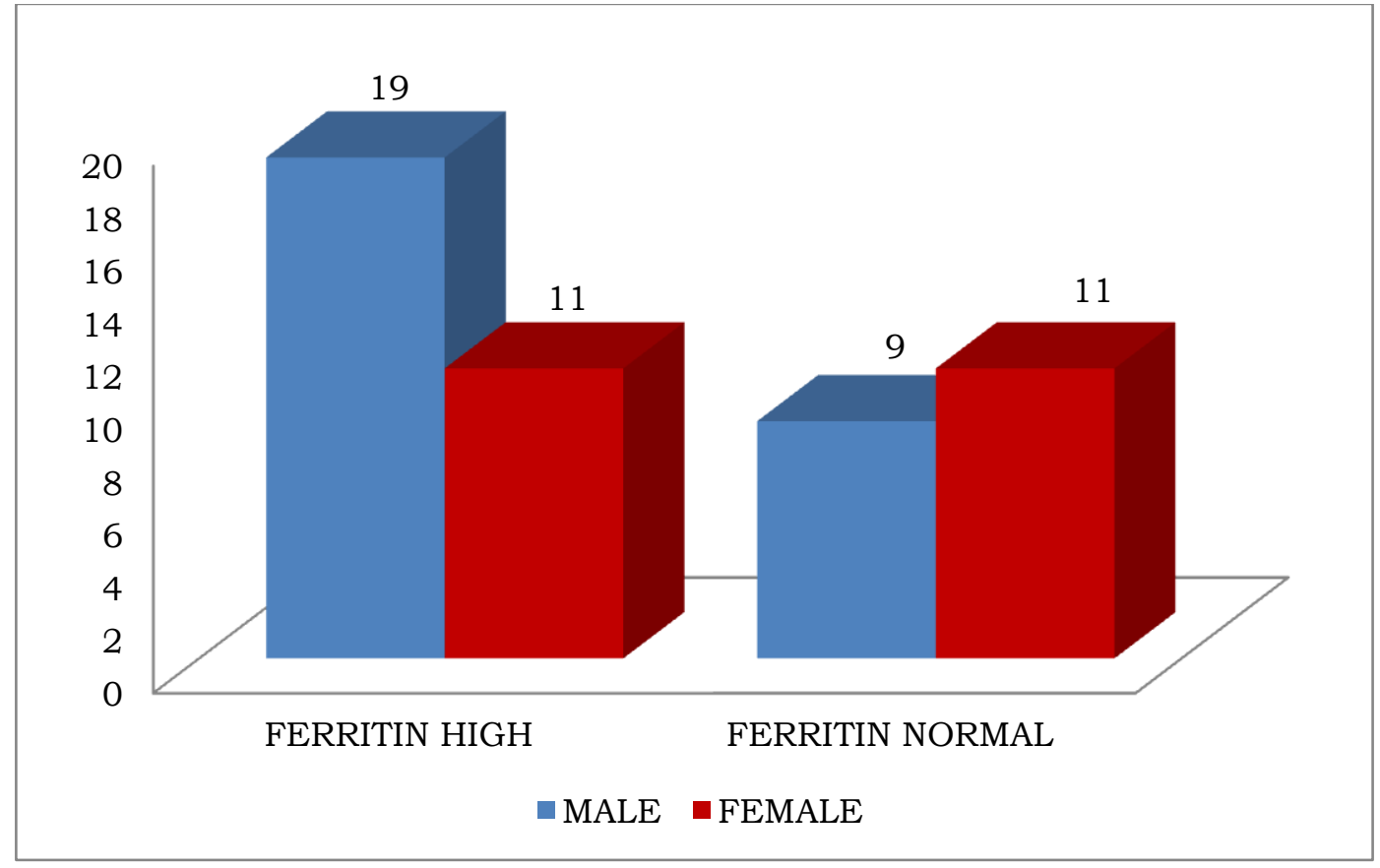


Table 4: Significance between Ferritin Levels and Thrombocytopenia

\begin{tabular}{|l|c|c|c|}
\hline \multirow{2}{*}{ FERRITIN } & \multicolumn{2}{|c|}{ THROMBOCYTOPENIA } & \multirow{2}{*}{ TOTAL } \\
\cline { 2 - 4 } & PRESENT & ABSENT & \\
\hline HIGH & 16 & 14 & 30 \\
\hline NORMAL & 3 & 17 & 20 \\
\hline TOTAL & 19 & 31 & 50 \\
\hline
\end{tabular}

Chi square test was applied to test significance between serum ferritin levels and thrombocytopenia. There was significant corelation $(\mathrm{p}=0.006)$ between serum ferritin levels and thrombocytopenia. This implies that increase in ferritin levels is associated with significant decrease in platelet count.

Figure 4: Significance between Ferritin Levels and Thrombocytopenia
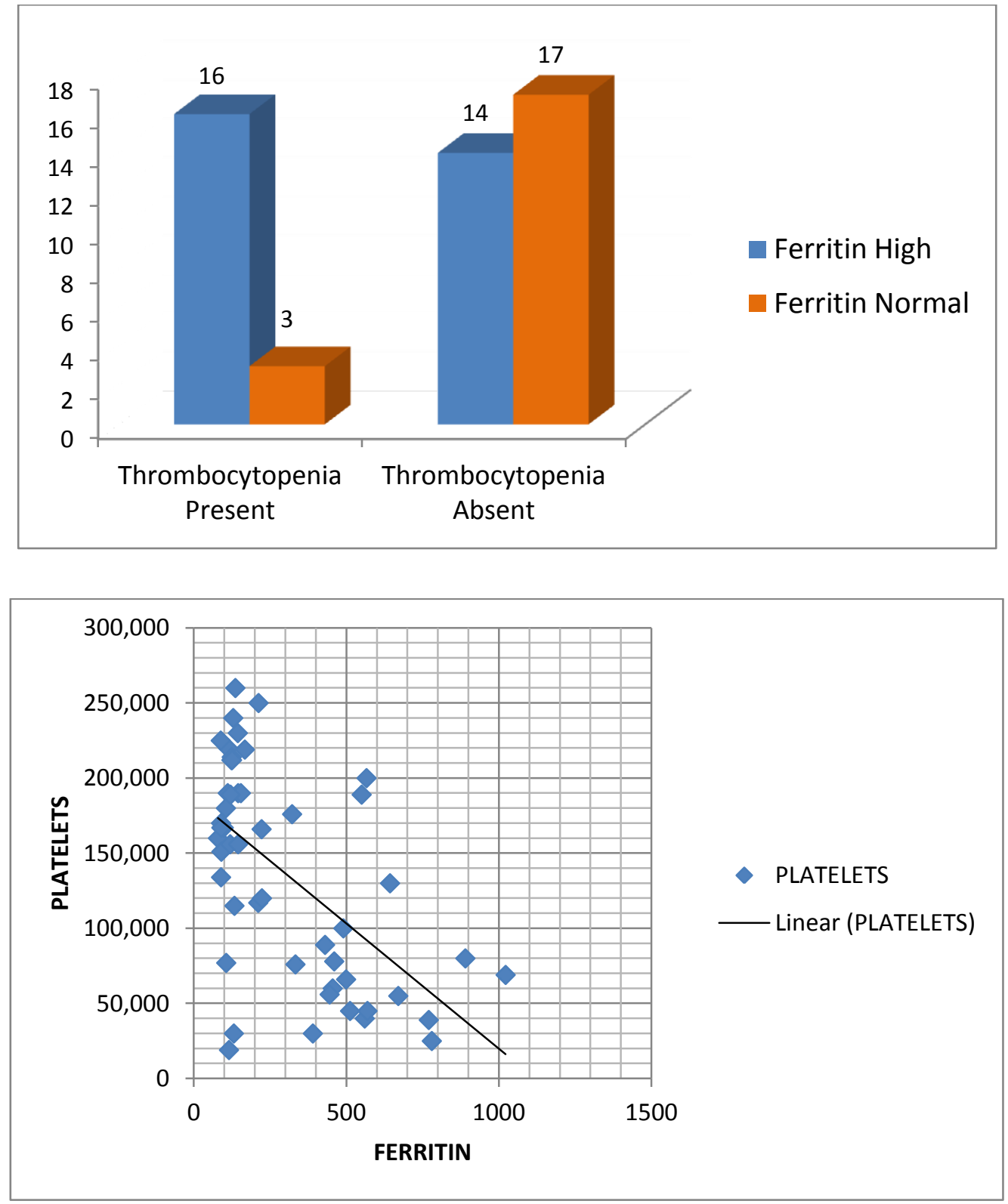

\section{Results and Analysis}

1) In this study 50 dengue $\operatorname{IgM}$ Elisa POSITIVE patients were studied.

2) Out of 50 patients, 28 were males and 22 were females.
3) Most of the patients were in the age group of 11-25 and least number of patients were in the age group of 56-70.

4) There was significant decrease in platelet count in patients with high serum ferritin levels. 


\section{Discussion}

Currently dengue is causing major public health concern throughout the World particularly in South East Asian countries. Serum ferritin levels are elevated in patients with dengue fever and elevated serum ferritin levels are associated with increased incidence of thrombocytopenia

In a study conducted by Petchaiappan $\mathrm{V}$ et al. serum ferritin levels negatively correlated with the platelet count $(\mathrm{r}=0.51, \mathrm{p}<0.001)$

Study conducted by Soundravally $\mathrm{R}$ et al. concluded that raised ferritin levels could predict the dengue severity with sensitivity of $76.9 \%$ and specificity of $83.3 \%$ on the day of admission.

Study conducted in Aruba and Brazil showed increased levels of serum ferritin were significantly associated with disease severity in dengue virus infection.

In a study conducted by Diwakar $\mathrm{TN}$ et al. concluded that significant association was found between raised serum ferritin, AST, ALT and severe Dengue $(\mathrm{p}<0.001)$

\section{Conclusion}

Hyperferritinemia can be used as a indicator for assessing disease severity and prognosis of dengue fever patients. Those patients with high serum ferritin levels should be monitored carefully than patients with normal serum ferritin levels

\section{References}

1. Hardcover, Fauci Anthony MD, Dennis Kasper, Stephen Hauser. Principles of Internal Medicine, Vol.2, Harrisons 19th Edition, Page 319.

2. Working to overcome the global impact of neglected tropical diseases [Internet]. www.who.int. 2010 [cited 30 March 2018]. Available from: http://whqlibdoc.who.int/publications/201 0/9789241564090_e ng.pdf

3. Back AT and Lundkvist A, Dengue viruses - an overview. Infect Ecol Epidemiol $2013 ; 3 ; 10-13$.
4. Wang SM. Sekaran SD, Early diagnosis of Dengue infection using a commercial dengue Duo rapid test kit for the detection of NSI, IgM, IgG, AM J tro Med Hyg 2010; 83: 690-95.

5. Samanta J and Sharma V, Dengue and its effects on liver. World J Clin cases 2015; $3 ; 125-31$.

6. Lee LK, Gan VC Lee VJ, Tan AS, Leo YS, Lye DC. Clinical relevance and discriminatory value of elevated liver aminotransferase levels of Dengue severity. Plos Negl Trop Dis 2012; 6; e1676.

7. Soundaravally R, Ageishkumar B, Daisy M, Sherin J, Cleetus CC, Ferritin levels predict sever dengue. Infection 2015; 43 ; 13-19.

8. Soumyabrata Roy chauduri et al. Serum ferritin a backstage weapon in Diagnosis of Dengue fever: Interdisciplinary perspectives on infectious diseases, Vol (2017), article Id : 7463489:

9. T. Ho. S. Wang, R. Anderson , C. Liu, Antibodies in dengue immunopathogenisis, Journal of Formosan Medical Association, Vol 112; no.1; pp 12; 2013.

10. Muhammad Nadeem et al, Serum Ferritin: an indicator of disease severity in patients with dengue infection. Jouranl of Rawalpindi Medical College (JRMC); 2016; 20(3); 165 - 167. 